\title{
Síndrome de DRESS por losartán
}

\section{Losartan-induced DRESS syndrome}

\author{
Diego Armando Oquendo-Gahona, Adriana Zamora-Suárez, Cindy Consuelo Romero-Méndez, \\ Juan Camilo Cifuentes-González • Neiva (Colombia)
}

DOI: https://doi.org/10.36104/amc.2021.2081

\section{Resumen}

Introducción: la reacción a fármacos con eosinofília y síntomas sistémicos conocido como síndrome DRESS corresponde a una reacción de hipersensibilidad medicamentosa, que puede llevar a la presentación de lesiones dermatológicas y compromiso de órganos internos. Clásicamente se ha asociado a una gran variedad de fármacos entre los cuales se incluyen anticonvulsivantes aromáticos, alopurinol y antibióticos como los principales responsables.

Objetivo: se presenta el caso de un paciente con síndrome de DRESS secundario a losartán manifestando síntomas cutáneos y compromiso hepático leve, fármaco para el cual hasta la fecha no existen reportes de presentar relación de causalidad con esta patología. Se instauró tratamiento de forma temprana incluyendo el retiro del medicamento responsable e inicio de corticoide sistémico oral observándose una adecuada respuesta

Conclusión: se debe estar atento a la aparición de lesiones dermatológicas con el uso de diferentes grupos de medicamentos, no sólo a los reportados clásicamente ya que cualquier fármaco podría ser potencial responsable de reacción de hipersensibilidad. (Acta Med Colomb 2021; 46. DOI: https://doi.org/10.36104/amc.2021.2081).

Palabras clave: síndrome de DRESS, losartán, hipersensibilidad farmacológica.

\section{Abstract}

Introduction: drug reactions with eosinophilia and systemic symptoms, known as DRESS syndrome, are a hypersensitivity reaction to medications which can lead to skin lesions and internal organ involvement. This syndrome has typically been associated with a wide variety of medications, including aromatic anticonvulsants, allopurinol and antibiotics as the main culprits.

Objective: we present the case of a patient with DRESS syndrome secondary to losartan, manifesting skin symptoms and mild hepatic involvement. Up until now, there have been no reports of losartan as the cause of this condition. Prompt treatment was instated including the withdrawal of the offending medication and initiation of oral systemic steroids, with a satisfactory response.

Conclusion: caregivers should be alert to the appearance of skin lesions with the use of different groups of medications, not just those typically reported, since any medication could potentially cause a hypersensitivity reaction. (Acta Med Colomb 2021; 46. DOI: https://doi.org/10.36104/amc.2021.2081).

Keywords: DRESS syndrome, losartan, drug hypersensitivity.
Dr. Diego Armando Oquendo-Gahona: Residente de Medicina Interna y Especialista en Epidemiología. Universidad Surcolombiana; Dra.Adriana Zamora-Suárez: Toxicóloga, Grupo desarrollo social, salud pública y derechos humanos. Docente Facultad de Medicina, Universidad Surcolombiana. Toxicóloga Hospital Universitario Hernando Moncaleano Perdomo; Dra.- Cindy Consuelo Romero-Méndez: Residente de Geriatría, Universidad Nacional de Colombia, Especialista en Epidemiología, Universidad Surcolombiana; Dr. Juan Camilo Cifuentes-González: Especialista en Medicina Interna. Universidad Surcolombiana.. Neiva (Colombia).

Correspondencia: Dr. Juan Camilo CifuentesGonzález. Neiva (Colombia)

E-Mail: chifu8600@gmail.com Recibido: 25/XII/2020 Aceptado: 6/IV/2021

\section{Introducción}

El síndrome de DRESS (Drug Reaction with Eosinophilia and Systemic Symptoms) o reacción a fármacos con eosinofilia y síntomas sistémicos, es una reacción de hipersensibilidad inducida por fármacos que puede tener un espectro de enfermedad leve hasta casos potencialmente mortales en donde se incluye anomalías cutáneas, hematológicas (eosinofilia, linfocitosis atípica), linfaadenopatía y afección de órganos internos como riñón, hígado y pulmón (1). Actualmente se recomienda que se mencione esta entidad como síndrome de hipersensibilidad inducido por fármacos/ reacción a fármacos con eosinofilia y síntomas sistémicos (DiHS/DRESS) (2).

La incidencia de ésta patología se ha reportado en 0.9-1 por cada 1000-10 000 exposiciones a fármacos $(3,4)$. En una revisión de la literatura en donde se incluyeron 131 artículos y 172 pacientes se encontró que los fármacos más frecuentemente asociados a esta patología fueron carbamazepina (27\%), alopurinol (11\%), lamotrigina (6\%), fenobarbital (6\%), sulfasalazina (6\%), fenitoína (4\%), 
abacavir (3\%) entre otros, con una media en tiempo de aparición posterior al inicio del fármaco de 3,6 semanas con un rango de 0.5 a 8 semanas (5).

\section{Fisiopatología}

Se han encontrado varias características que demuestran que se trata de una reacción de hipersensibilidad, como son su reproducibilidad con pruebas cutáneas, la reaparición de la enfermedad al haber una nueva exposición al medicamento desencadenante, y la necesidad de sensibilización previa (6).

Los criterios diagnósticos que se han planteado para esta entidad son los recomendados por RegiSCAR: 1) Rash agudo, 2) Reacción relacionada a fármaco, 3) Hospitalización, 4) Fiebre $>38^{\circ}$ 5) Alteraciones en laboratorio (al menos uno): linfocitosis ( $>4000)$ o linfopenia $<1500)$, trombocitopenia $(<100000)$, eosinofília $(>700), 6)$ Compromiso de $>1$ órgano interno y 7) Adenomegalia en $>2$ sitios. Siendo los tres primeros necesarios para el diagnóstico sumado a tres de los siguientes cuatro.

Se considera importante el reporte de este caso, ya que se trata de una patología poco frecuente, que se presenta generalmente tras la administración de fármacos anticonvulsivantes, pero que en Colombia no se tienen datos de esta entidad asociada a losartán, con la relevancia de que es un medicamento que se formula diariamente para el manejo de la hipertensión arterial.

\section{Presentación del caso}

Paciente masculino de 62 años de edad quien ingresa al servicio de urgencias por cuadro clínico de 10 días de evolución consistente en aparición de lesiones eritematosas, descamativas pruriginosas que se manifiestan en forma generalizada comprometiendo cara, tórax, abdomen y extremidades (Figura 1). En la revisión por sistemas menciona que no ha presentado síntomas catarrales, no fiebre, no dolor articular, tiene antecedente de hipertensión arterial para lo cual recibe losartán $50 \mathrm{mg} /$ vía oral cada 12 horas desde hace un mes, previamente recibía enalapril, pero por episodios de tos aislados se realizó el cambio. No presenta otros antecedentes de importancia. Al momento del ingreso presentaba signos vitales: Tensión arterial: 130/80, frecuencia cardiaca: 80 latidos por minutos, frecuencia respiratoria: 16 respiraciones por minuto, saturación: 99\%. Como hallazgo positivo se encontró eritema morbiliforme localizado en miembros inferiores y tronco además lesiones eritematosas, costrosas localizadas en región mandibular, orejas, dorso y región posterior de miembros inferiores, se tomaron laboratorios (Tabla 1), en donde se evidencia presencia de eosinofilia, así como

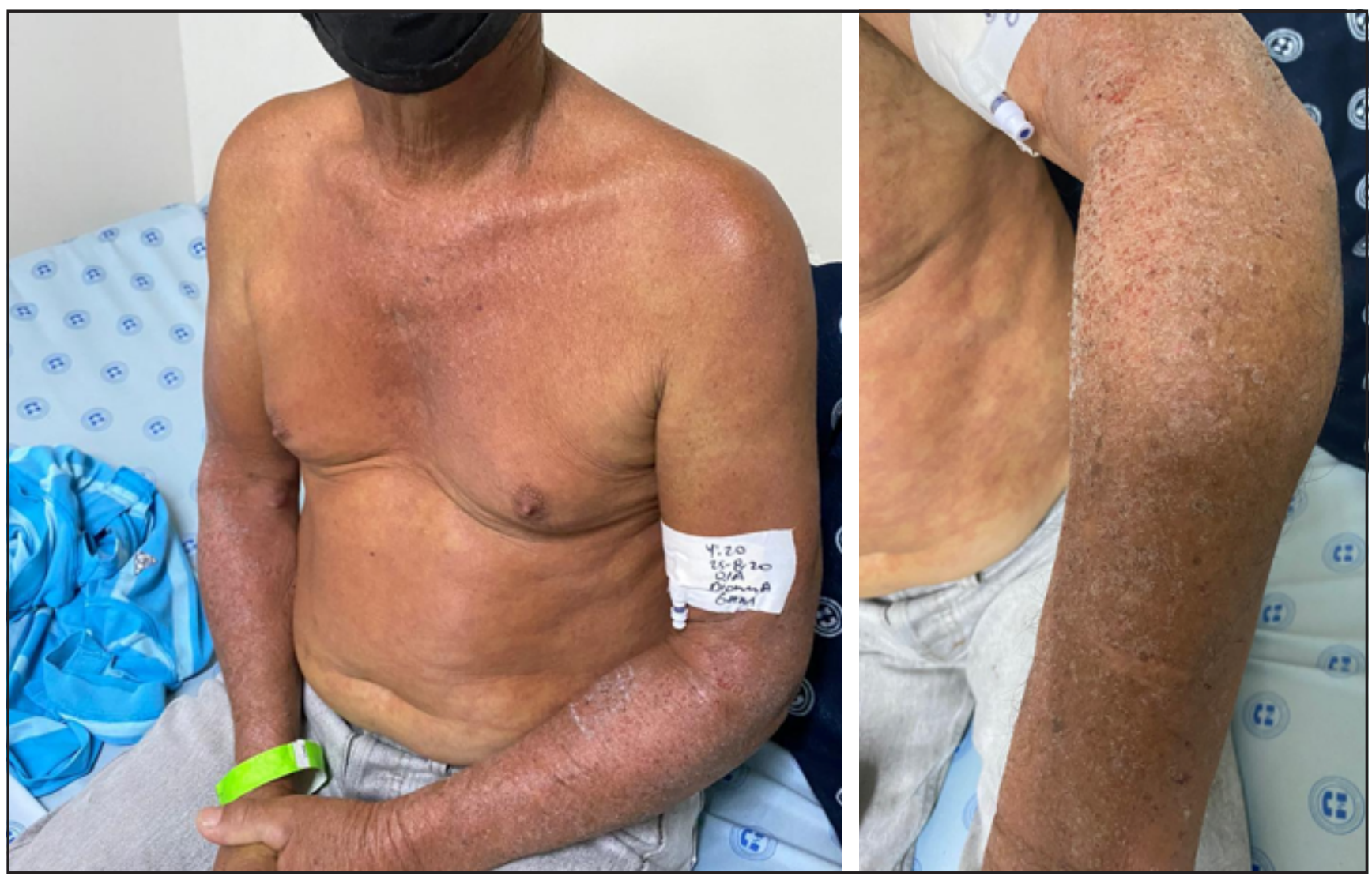

Figura 1. Lesiones eritematosas, descamativas pruriginosas que aparecen de forma generalizada comprometiendo cara, tórax, abdomen y extremidades. 
Tabla 1. Registro de laboratorios.

\begin{tabular}{|l|c|c|}
\hline & Día 1 & Día 6 (control) \\
\hline Leucocitos & $9550 / \mathrm{ul}$ & $6050 / \mathrm{ul}$ \\
\hline Neutrófilos & $5190 / \mathrm{ul}$ & $4310 / \mathrm{ul}$ \\
\hline Linfocitos & $1120 / \mathrm{ul}$ & $850 / \mathrm{ul}$ \\
\hline Eosinófilos & $2640 / \mathrm{ul}$ & $370 / \mathrm{ul}$ \\
\hline Basófilos & $10 / \mathrm{ul}$ & $10 / \mathrm{ul}$ \\
\hline Hemoglobina & $13.6 \mathrm{~g} / \mathrm{dl}$ & $12.9 \mathrm{~g} / \mathrm{dl}$ \\
\hline Plaquetas & $240 \mathrm{000} / \mathrm{ul}$ & $182 \mathrm{000} / \mathrm{ul}$ \\
\hline Aspartato aminotransferasa & $116 \mathrm{U} / \mathrm{l}$ & $45 / \mathrm{ul}$ \\
\hline Alanino aminotransferasa & $198 \mathrm{U} / \mathrm{l}$ & $127 / \mathrm{ul}$ \\
\hline Deshidrogenasa láctica & $507 \mathrm{u} / \mathrm{l}$ & $269 / \mathrm{ul}$ \\
\hline VSG & $8 \mathrm{~mm} / \mathrm{hora}$ & \\
\hline PCR & $3.81 \mathrm{mg} / \mathrm{dl}$ & \\
\hline Nitrógeno ureico & $8.2 \mathrm{mg} / \mathrm{dl}$ & \\
\hline Creatinina & $0.8 \mathrm{mg} / \mathrm{dl}$ & \\
\hline VIH & Negativo & \\
\hline VDRL & No reactivo & \\
\hline
\end{tabular}

pruebas de función hepáticas y LDH aumentadas. Se ingresa bajo la sospecha de síndrome de DRESS. Teniendo en cuenta que estas lesiones aparecieron posterior al cambio, en la medicación se indica suspender el losartán e iniciar terapia corticoide con posterior mejoría de las lesiones, se tomaron paraclínicos de control en los cuales se evidencia disminución de los eosinófilos y transaminasas. Se realizó biopsia de piel (Figura 2), con lo cual se confirma el diagnóstico de síndrome de DRESS por losartán.

\section{Discusión}

Cuando se habla de síndrome de DiHS/DRESS, se piensa en una reacción de hipersensibilidad asociado a medicamentos principalmente anticonvulsivantes, ya que históricamente éstos son los fármacos más comúnmente reportados como etiología de esta entidad. Se debe tener en cuenta que diferentes cohortes y reportes de caso han venido reportando diferentes grupos farmacológicos como nuevas etiologías, es así como en el 2011 en un estudio de revisión de la literatura se reportaron 44 medicamentos responsables, dentro de los cuales solo uno correspondía a un agente antihipertensivo siendo éste el captopril (5). En el registro RegiSCAR se encontraron 19 agentes etiológicos dentro de los cuales no hubo antihipertensivos (7). Aparte de los fármacos anticonvulsivantes destacan además alopurinol, antibióticos, fármacos antituberculosos y $\operatorname{AINES}(7,8)$.

Un punto importante a destacar en esta patología es la temporalidad, en nuestro paciente las lesiones iniciaron 20 días posterior a el cambio de tratamiento farmacológico. Se ha visto que la latencia (tiempo de inicio del medicamento a inicio de los síntomas) puede cambiar de acuerdo con el grupo farmacológico, siendo para la carbamazepina 33 días, alopurinol 30 días, antituberculosos 27 días, vancomicina 20 días, AINES 18 días, cefalosporinas 15 días (8). La temporalidad también se ha utilizado para considerar que un síndrome de DRESS es poco probable, si el inicio del medicamento fue hace más de tres meses, haberlo suspendido en un tiempo mayor a 14 días o iniciado en menos de tres días (7).

La presentación clínica es variable, iniciando en ocasiones con un síndrome febril el cual puede estar presente en $50-100 \%$ de los pacientes. Acompañarse de un pródromo
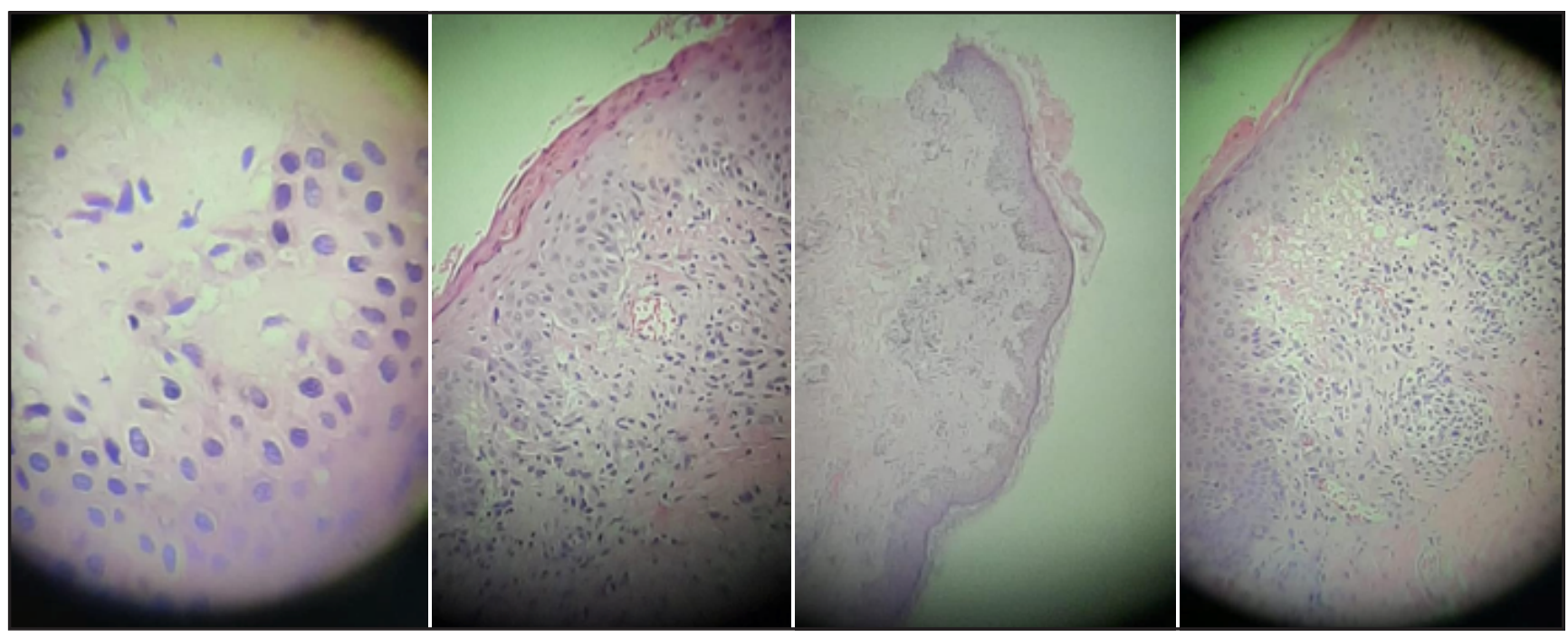

Figura 2. Biopsia de piel de tórax y antebrazo. Presencia de infiltrado linfocitario perivascular superficial de linfocitos asociado a cambio vascular de la basal asociado a la presencia de histiocitos, plasmocitos y eosinófilos dispersos perivasculares e intersticiales con edema de la dermis papilar, hay espongiosis, leve acantosis con ortoqueratosis en maya y focos de paraqueratosis. Hallazgos compatibles con Erupción morbiliforme por medicamentos. 
sugestivo de faringitis que evoluciona al rash cutáneo generalizado, macular, morbiliforme o en diana que luego se tornan confluentes y pruriginoso. Dentro de las lesiones dermatológicas, el rash maculopapular se presenta en 91$100 \%$ de los casos, mientras que el compromiso vesicular, mucoso y descamativo se presenta en menos de $30 \%$. Las adenopatías pueden presentarse en 4-60\% (8). En ocasiones suele haber una recaída de tres a cuatro días posterior a la suspensión del medicamento culpable, lo que confunde al clínico con un evento infeccioso, provocando inicio de tratamiento antibiótico no necesario que incluso genera reacciones cruzadas y empeora el cuadro. Debido a lo anterior se han utilizado reactantes de fase aguda como la procalcitonina y la proteína $\mathrm{C}$ reactiva (PCR) para diferenciar la infección bacteriana del síndrome de DRESS, encontrando que en pacientes con procesos infecciosos concomitantes tienen niveles de reactantes elevados, tomando puntos de corte para procalcitonina de $1.33 \mathrm{ng} /$ $\mathrm{ml}$ y PCR $10.83 \mathrm{ng} / \mathrm{ml}$ (9), siendo esto importante para el clínico a la hora de tomar decisiones sobre todo en cuanto a terapias antimicrobianas se refiere.

Cuando se enfrenta a un paciente con un posible síndrome de DiHS/DRESS, como primera medida se evalúa el probable fármaco culpable. En nuestro caso nos enfrentamos a un paciente que hace siete meses venía recibiendo enalapril, el cual se cambió a losartán por pobre tolerancia al primero. Se resalta que hasta en $80 \%$ de los casos se encuentra el fármaco culpable, pero el $20 \%$ restante no hay un fármaco claro, sobre todo cuando el paciente recibe múltiples medicamentos al tiempo. Se han propuesto diferentes métodos para determinar la probabilidad diagnóstica. Por lo cual se han planteado criterios diagnósticos tanto para DiHS y DRESS (previamente mencionado), aunque RegiSCAR tiene un sistema de puntuación propio para definir los casos en: definido, probable, posible y exclusión de caso (7). Son importantes los hallazgos paraclínicos dentro de lo cual resalta la presencia de eosinofília (95\% de los casos), leucocitosis (95\%), linfocitos atípicos $(35 \%)(5,7)$ y en algunos casos elevación de transaminasas (10). Como se ha mencionado previamente, se ha observado una relación de la enfermedad con la reactivación de patologías virales, como herpes virus humano (HHV) -6, HHV-7, el virus de Epstein-Barr (EBV) y citomegalovirus. Se cree que la presencia de la reactivación viral puede estar en relación a cuadros clínicos más severos, compromiso orgánico y curso de la enfermedad más prolongado (11). Aunque se ha visto una fuerte asociación con HHV-6, la presencia de éste no es necesario para el diagnóstico de la enfermedad. Las lesiones de órganos internos incluyen compromiso hepático con elevación de pruebas de función hepática e hiperbilirruminemia, a nivel renal elevación de la creatinina sérica, disminución de la tasa de filtración glomerular, hematuria y alteraciones del sedimento urinario, incluso la necesidad de terapia de remplazo renal, puede ocurrir también compromiso pulmonar, gastrointestinal y cardiaco (7).
Para el tratamiento de la enfermedad no se cuentan con ensayos clínicos que indiquen cuál es la mejor evidencia. El primer paso es suspender el fármaco culpable de la enfermedad, luego se puede elegir tratamiento corticoide tópico en casos no graves, o en donde no se documente compromiso de órganos internos, este manejo puede ser con betametasona propionato o clobetasol propionato, aplicado una o dos veces al día (12). En el 2019 Mizukawa et al (13) proponen una escala para determinar la severidad de la enfermedad y predecir la reactivación de la infección por citomegalovirus (Tabla 2, Figura 3), se realiza la sumatoria siendo leve si $\leq 0$, moderado si 1-3 puntos, severo $\geq 4$ puntos. Si lo aplicamos a nuestro paciente el paciente cumple con lesiones erosivas que comprometían 10-29\% de la superficie corporal total, lo que puntúa como 1 y clasifica la enfermedad como moderada por lo cual se inició tratamiento con corticoide sistémico oral, observando una resolución de su sintomatología además de mejoría en el reporte de paraclínicos (Tabla 1). se han probado otros tratamientos a base de inmunoglobulina, aunque al parecer se ha encontrado una relación en el uso de inmunoglobulina y la aparición de síndrome poliglandular tipo III en pacientes en edad pediátrica (14).

\section{Conclusión}

El DiHS/DRESS es una enfermedad que se ha asociado a múltiples fármacos, presentando algunos de ellos mayor relación con la aparición de la enfermedad. En este caso

Tabla 2. Escala para evaluar la severidad del síndrome de hipersensibilidad inducida por fármacos y predicción de los resultados de la enfermedad.

\begin{tabular}{|c|c|c|c|c|}
\hline Parámetros & -1 & 1 & 2 & 3 \\
\hline \multicolumn{5}{|l|}{ Parámetros fijos } \\
\hline Edad (años) & $\leq 40$ & & $\geq 75$ & \\
\hline $\begin{array}{l}\text { Duración de la exposición al } \\
\text { fármaco después del inicio (días) }\end{array}$ & & $\geq 7$ & & \\
\hline Exposición a alopurinol & & si & & \\
\hline \multicolumn{5}{|l|}{ Parámetros variables } \\
\hline Uso de prednisolona & & & Pulso & \\
\hline \multicolumn{5}{|l|}{ Compromiso de piel } \\
\hline $\begin{array}{l}\text { Rash eritematoso } \\
\text { (\% de área corporal total) }\end{array}$ & & $\geq 70 \%$ & Eritrodermia & \\
\hline $\begin{array}{l}\text { Lesiones erosivas } \\
\text { (\% de área corporal total) }\end{array}$ & & $10-29 \%$ & & $\geq 30 \%$ \\
\hline Fiebre $>38.5^{\circ}$ (días) & & $2-6$ & $\geq 7$ & \\
\hline $\begin{array}{l}\text { Pérdida de apetito en días } \\
\text { (consumo } \leq 70 \% \text { de alimentación } \\
\text { normal }\end{array}$ & & $\geq 5$ & & \\
\hline $\begin{array}{l}\text { Disfunción renal } \\
\text { (creatinina, } \mathrm{mg} / \mathrm{dl})\end{array}$ & & $1-2$ & & $>2$ o HD \\
\hline Disfunción hepática (ALT, UI/l) & & $400-1000$ & & $>1000$ \\
\hline Proteína C reactiva (mg/dl) & $\leq 2$ & $10-15$ & $>15$ & \\
\hline \multicolumn{5}{|c|}{$\begin{array}{l}\text { ALT: alanino aminotransferasa, HD: hemodiálisis. Los parámetros variables se pueden } \\
\text { medir de forma temprana ( } 0-3 \text { días después de la presentación inicial) y de forma tardía } \\
\text { ( } 2-4 \text { semanas después de la presentación inicial). }\end{array}$} \\
\hline
\end{tabular}




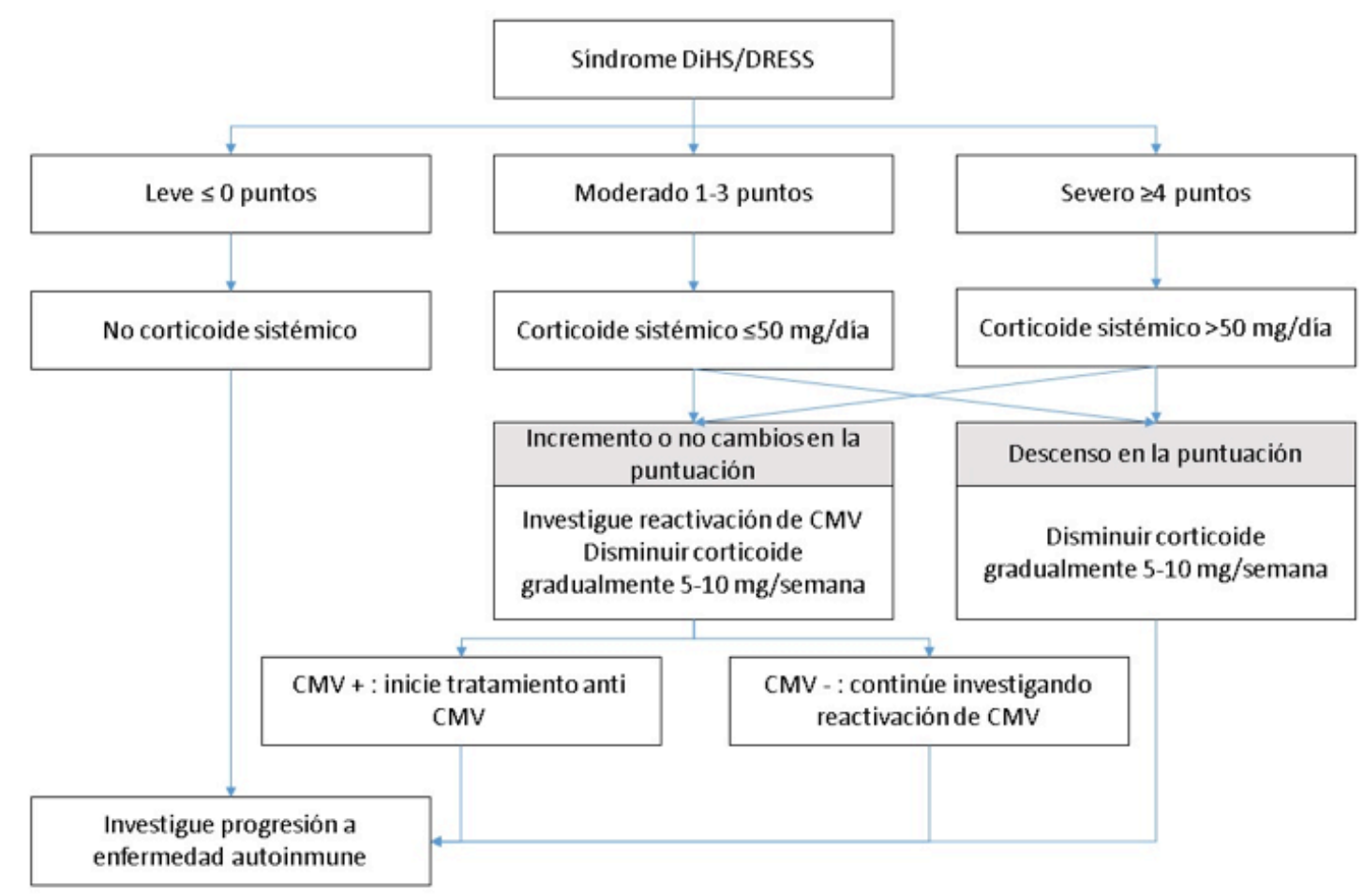

CMV: citomegalovirus; DiHS / DRESS: síndrome de hipersensibilidad inducida por fármaco/reacción farmacológica con eosinofilia y síntomas sistémicos.

Figura 3. Diagrama de flujo propuesto para el diagnóstico y la predicción de resultados del síndrome de hipersensibilidad inducida por fármacos.

reportamos esta patología asociada a un fármaco que a la fecha no hemos tenido conocimiento de tener relación de causalidad, por lo cual resaltamos la necesidad de estar atentos a las lesiones dermatológicas y realizar una revisión detallada de la medicación recibida por el paciente, ya que un retiro de la medicación asociado a un tratamiento temprano, puede llevar a resultados y pronósticos favorables.

\section{Referencias}

1. Bocquet H, Bagot M, Roujeau JC. Drug-induced pseudolymphoma and drug hypersensitivity syndrome (Drug Rash with Eosinophilia and Systemic Symptoms: DRESS). Semin Cutan Med Surg. 1996 Dec;15(4):250-7.

2. Shiohara T, Mizukawa Y. Drug-induced hypersensitivity syndrome (DiHS)/drug reaction with eosinophilia and systemic symptoms (DRESS): An update in 2019. Allergol Int. 2019 Jul;68(3):301-8.

3. Fiszenson-Albala F, Auzerie V, Mahe E, Farinotti R, Durand-Stocco C, Crickx B, et al. A 6-month prospective survey of cutaneous drug reactions in a hospital setting. Br J Dermatol. 2003 Nov; 149(5):1018-22.

4. Muller P, Dubreil P, Mahé A, Lamaury I, Salzer B, Deloumeaux J, et al. Drug Hypersensitivity Syndrome in a West-Indian population. Eur J Dermatol. 2003;13(5):478-81.

5. Cacoub P, Musette P, Descamps V, Meyer O, Speirs C, Finzi L, et al. The DRESS syndrome: a literature review. Am J Med. 2011 Jul;124(7):588-97.

6. Cardona, M; Galindo,LF; Díaz-Guío. Síndromes de hipersensibilidad inducidos por medicamentos en las unidades de cuidados intensivos. Rev Asoc Colomb Dermatol. 2018; 26 : 3 (julio-septiembre):170-83.

7. Kardaun SH, Sekula P, Valeyrie-Allanore L, Liss Y, Chu CY, Creamer D, et al. Drug reaction with eosinophilia and systemic symptoms (DRESS): an original multisystem adverse drug reaction. Results from the prospective RegiSCAR study. Br J Dermatol. 2013 Nov; 169(5):1071-80.

8. Sim DW, Yu JE, Jeong J, Jung J-W, Kang H-R, Kang DY, et al. Variation of clinical manifestations according to culprit drugs in DRESS syndrome. Pharmacoepidemiol Drug Saf. 2019 Jun;28(6):840-8.

9. Rhyou H-I, Nam Y-H. Procalcitonin as a diagnostic marker for differentiating DRESS syndrome from bacterial infection. Vol. 123, Annals of allergy, asthma \& immunology : official publication of the American College of Allergy, Asthma, \& Immunology. United States; 2019. p. 307-309.e1.

10. Ramírez-Cornejo C, Downey-Saldivia C, Del Puerto-Troncoso C, Del Barrio- Diaz P, Vera-Kellet C, Andino-Navarrete R. Variables clínicas, de laboratorio e histopatológicas en Síndrome de hipersensibilidad o erupción por drogas con eosinofilia y síntomas sistémicos (DRESS). Rev Chil Dermatología. 2019;34(4):114-8.

11. Tohyama M, Hashimoto K, Yasukawa M, Kimura H, Horikawa T, Nakajima K, et al. Association of human herpesvirus 6 reactivation with the flaring and severity of drug-induced hypersensitivity syndrome. Br J Dermatol. 2007 Nov;157(5):934-40.

12. Funck-Brentano E, Duong T-A, Bouvresse S, Bagot M, Wolkenstein P, Roujeau J-C, et al. Therapeutic management of DRESS: a retrospective study of 38 cases. J Am Acad Dermatol. 2015 Feb;72(2):246-52.

13. Mizukawa Y, Hirahara K, Kano Y, Shiohara T. Drug-induced hypersensitivity syndrome/drug reaction with eosinophilia and systemic symptoms severity score: A useful tool for assessing disease severity and predicting fatal cytomegalovirus disease. J Am Acad Dermatol. 2019 Mar;80(3):670-678.e2.

14. Morita C, Yanase T, Shiohara T, Aoyama Y. Aggressive treatment in paediatric or young patients with drug-induced hypersensitivity syndrome (DiHS)/drug reaction with eosinophilia and systemic symptoms (DRESS) is associated with future development of type III polyglandular autoimmune syndrome. BMJ Case Rep. 2018 Oct;2018:bcr2018225528.

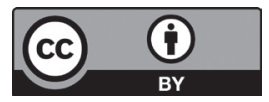

